\title{
Synthèse
}

Dynamiques des filières et secteurs

\section{Analyse comparée de la dynamique de la production laitière dans les pays du Maghreb}

Mohamed Taher Sraïri ${ }^{1}$

Mondher Ben Salem²

Alain Bourbouze ${ }^{3}$

Mohammed Elloumi ${ }^{4}$

Bernard Faye ${ }^{5}$

Taoufik Madani ${ }^{6}$

Hacène Yakhlef ${ }^{7}$

${ }^{1}$ Institut agronomique

et vétérinaire Hassan II (IAV Hassan II),

Département des productions animales,

BP 6202,

Madinate Al Irfane,

10101 Rabat

Maroc

$<$ mt.srairi@iav.ac.ma>

2 Institut national de la recherche agronomique (Inrat),

Laboratoire des productions animales

et fourragères,

Rue Hédi Karray,

2049 Ariana

Tunisie

<lahmar.mondher@iresa.agrinet.tn>

${ }^{3}$ Institut agronomique méditerranéen

de Montpellier (lamm),

3191, route de Mende,

34090 Montpellier

<bourbouze@iamm.fr>

4 Institut national de la recherche agronomique

(Inrat),

Laboratoire d'économie rurale

et d'analyse des données,

Rue Hédi Karray,

2049 Ariana

Tunisie

<elloumi.mohamed@iresa.agrinet.tn>

${ }^{5}$ Centre de coopération internationale

en recherche agronomique

pour le développement (Cirad),

Département Élevage et médecine vétérinaire

tropicale (Emvt)

Campus de Baillarguet,

TA 30/A

34398 Montpellier cedex 5

<bernard.faye@cirad.fr>

${ }^{6}$ Université Ferhat-Abbas,

Faculté des sciences,

Département d'agronomie,

Sétif 19000

Algérie

<madani2000dz@yahoo.fr>

7 Institut national agronomique d'El Harrach,

Département de zootechnie

16200 Hacène Badi,

El Harrach,

Alger

Algérie

<zootech@ina.dz>

Tirés à part : M.T. Sraïri

\begin{abstract}
Résumé
L'élevage bovin laitier a été retenu par les pouvoirs publics des pays du Maghreb comme un axe important des politiques agricoles post-indépendance, pour la création d'emplois et de revenus et pour assurer l'approvisionnement en protéines animales de populations en plein essor démographique avec des habitudes alimentaires changeantes. Les politiques laitières ont connu des évolutions divergentes par pays (approvisionnement avec de la poudre de lait importé, en Algérie ; production bovine locale, au Maroc; et voie intermédiaire, en Tunisie), avec des instruments variables, ce qui a généré des performances des filières contrastées. Cet article fait le point sur les évolutions récentes de la production bovine laitière dans les pays du Maghreb ainsi que sur ses perspectives : défis posés par la continuation des efforts de sécurisation de l'approvisionnement et mise à niveau des filières par une meilleure maîtrise de la qualité des produits et de la répartition des revenus entre les opérateurs.
\end{abstract}

Mots clés : bovin laitier ; industrie laitière ; Maghreb ; production laitière.

Thèmes : productions animales ; économie et développement rural ; territoire, foncier, politique agricole.

\section{Abstract \\ Comparative analysis of the dynamics of dairy production in the Maghreb countries}

Dairy cattle production has always been considered as an important axis of all agricultural policies adopted by Maghreb countries since the beginning of the post Independence era (Algeria, Morocco, and Tunisia). It has mainly been encouraged as a means of securing income and employment at the farm level. It is also considered to be a relevant way of ensuring the supply of high quality protein for rapidly growing populations characterised by changing nutritional habits. Dairy policies vary from country to country (supply with imported milk in Algeria, local dairy cattle production in Morocco and an intermediary choice in Tunisia), relying on different approaches and producing contrasting results. Thus, obvious differences exist between countries in terms of dairy performance and domestic market supply. This paper aims at comparing the recent dynamics of dairy production in the Maghreb countries with an emphasis on their future prospects: challenges due to the continuing effort to secure supply and to the upgrading of the whole supply chain by a better management of dairy goods quality and an equitable distribution of the income to dairy operators.

Key words: dairy cattle; dairy industry; Maghreb; milk production.

Subjects: animal productions; economy and rural development; territory, agricultural land use, agricultural policy. 
l'Indépendance des trois pays du Maghreb (Algérie, Maroc et Tunisie), au début de la seconde moitié du $\mathrm{Xx}^{\mathrm{e}}$ siècle, les autorités ont été confrontées à une demande croissante en protéines animales de la part de populations en plein essor et qui s'urbanisaient rapidement. Étant donné la valeur symbolique que les Maghrébins accordent au lait (accueil des invités et accompagnement de régimes alimentaires dominés par les céréales), un effort impérieux devait être mené pour sécuriser son approvisionnement. Des politiques de développement de l'élevage se mirent en place, et se focalisèrent sur le lait de vache (Projet Sebou, 1961 ; Rondia et al., 1985). En effet, à la différence des pays du Machrek, il n'existe pas au Maghreb de traditions de traite des petits ruminants, et le lait consommé provient essentiellement des vaches. La production et la consommation de lait camelin demeurent anecdotiques, à l'exception des villes les plus méridionales (Faye et al., 1998).

Au Maghreb, les bovins ont toujours été l'espèce dominante des zones les plus fertiles (bour favorable, piémonts et terres irriguées). Ils assurent une meilleure efficacité de la transformation des fourrages (Spedding, 1979), valorisent les sousproduits de l'agriculture récupérés soigneusement sur le terroir, et prodiguent un revenu régulier en occupant une main-d'œuvre abondante. Ce dernier aspect est fondamental à considérer, étant donné le niveau de sous-emploi, véritable fléau social (Abaab et al., 1995). Nonobstant ces avantages, l'élevage bovin et l'approvisionnement des marchés en lait ont connu des évolutions divergentes, en raison des politiques adoptées et des instruments mis en place. Avec un recul de plus de trente années, au vu des performances réalisées, les avantages et les inconvénients des choix opérés peuvent être, à présent, étudiés. Dans cet article, après avoir rappelé le cadre naturel de l'élevage bovin au Maghreb, les politiques laitières adoptées et leurs instruments seront inventoriés pour chacun des trois pays. Puis, les performances des filières laitières et leurs dynamiques récentes seront analysées. Finalement, une analyse prospective des filières laitières maghrébines sera ébauchée.

\section{Le cadre naturel de l'élevage bovin dans les pays du Maghreb}

Le Maghreb est marqué par de hautes terres, les Atlas, qui s'abaissent progressivement vers l'est. Le relief est un élément d'explication des différences régionales (Balta, 1990), mais le climat est plus déterminant pour saisir l'évolution des systèmes agraires, constituant le facteur décisif des niveaux de production. Étant donné les larges variations intra- et interannuelles du climat, les résultats du secteur agricole sont très contrastés d'une année sur l'autre.

Au niveau démographique, les trois pays du Maghreb se sont caractérisés par le doublement de leurs populations de 1970 à l'an 2000. Pour faire face à ces évolutions, l'intensification de l'agriculture s'est appuyée sur des programmes d'irrigation qui concernent aujourd'hui près de 1,8

\section{Tableau 1. Structure génétique du cheptel bovin et importations de génisses laitières dans les pays du Maghreb (chiffre de 1999, avant l'interdiction des importations de bétail).}

Table 1. Genetic structure of herds and heifer imports in the Maghreb countries (figures of 1999, before the ban on imports).

\begin{tabular}{|c|c|c|c|}
\hline & Algérie & Maroc & Tunisie \\
\hline Effectif total de vaches & 675000 & 1150000 & 475000 \\
\hline $\begin{array}{l}\text { Effectif de vaches de races } \\
\text { améliorées (dites « pures ») }\end{array}$ & 130000 & 200000 & 194000 \\
\hline $\begin{array}{l}\text { Effectif de vaches importées } \\
\text { depuis } 1975\end{array}$ & 120000 & 275000 & 90000 \\
\hline $\begin{array}{l}\text { Taux de races améliorées } \\
\text { "pures » (croisés non comptés) } \\
(\%)\end{array}$ & 19 & 17 & 35 \\
\hline
\end{tabular}

million d'hectares : 450000 en Algérie, 1 million au Maroc et 350000 en Tunisie. Dans les régions bénéficiant d'une pluviométrie clémente, des barrages ont été aménagés pour stocker les précipitations et pallier leur irrégularité. Ces conditions climatiques représentent une contrainte majeure pour l'élevage et accentuent sa vulnérabilité. Bien que la transhumance et le nomadisme aient naguère atténué l'effet des sécheresses, l'intensification de l'élevage ne peut plus en aucun cas s'en contenter. Aussi, une promotion d'envergure des cultures fourragères, a-t-elle été adoptée, surtout dans les périmètres irrigués. Dans les zones d'agriculture pluviale, des espèces traditionnelles (vesce avoine, pois, et lupin), voire nouvelles (betterave, sorgho...) ont été encouragées (Guessous, 1991; Jaritz et Bounejmate, 1995). En parallèle, la structure génétique du cheptel bovin a été radicalement modifiée. Les pouvoirs publics, pressés par le temps, ont orienté en priorité leurs efforts vers la création d'un noyau de vaches de type pie noir (tableau 1). Par conséquent, les croisements avec les souches locales se sont multipliés, à travers l'insémination artificielle, mais souvent de façon non planifiée (Djemali et Berger, 1992).

\section{Les politiques laitières et leurs instruments dans les trois pays du Maghreb}

Les plans agricoles qui se sont succédé dans les trois pays ont fait la part belle à deux types de produits : les aliments de première nécessité (céréales, sucre, huiles, viande et lait) et les denrées d'exportation (primeurs, fruits et légumes, vin) (El Khyari, 1985). Pour les produits vivriers de base, les bilans de ces mesures demeurent mitigés (Bencharif et al., 1996 ; Talha, 1994). De surcroît, la situation est loin d'être homogène pour les trois pays.

En Algérie, les politiques ont d'abord privilégié l'industrialisation par rapport à l'agriculture. Aussi, l'État s'est-il engagé à assurer, via le marché international, l'approvisionnement de la population (Dufumier, 1996). L'Algérie demeure encore un des principaux importateurs mondiaux de lait (Chalmin, 1999) : huit 
Tableau 2. Évolution récente des niveaux d'importation en lait dans les pays du Maghreb (d'après Food and Agriculture Organisation, 2003).

Table 2. Recent evolution in milk import levels in the Maghreb countries (from FAO, 2003).

\begin{tabular}{|c|c|c|c|c|c|c|}
\hline \multirow[t]{2}{*}{ Année } & \multicolumn{2}{|c|}{ Algérie } & \multicolumn{2}{|c|}{ Maroc } & \multicolumn{2}{|c|}{ Tunisie } \\
\hline & t éq. lait & kg/hab.an & t éq. lait & kg/hab.an & t éq. lait & kg/hab.an \\
\hline 1992 & 1721437 & 66,2 & 298319 & 12,6 & 236742 & 24,2 \\
\hline 1994 & 1880468 & 72,3 & 311327 & 12,9 & 142976 & 15,0 \\
\hline 1996 & 1618486 & 62,7 & 301432 & 12,3 & 145674 & 15,4 \\
\hline 1998 & 1786790 & 65,5 & 209262 & 9,4 & 72089 & 7,5 \\
\hline 2000 & 1814625 & 66,0 & 245256 & 10,2 & 63125 & 6,4 \\
\hline 2002 & 1765482 & 65,1 & 250145 & 10,4 & 71452 & 7,0 \\
\hline
\end{tabular}

t éq. lait : tonne équivalent lait. fois plus que le Maroc (tableau 2). Malgré les ressources du pays, la production bovine laitière locale a été négligée (Bourbouze et al., 1989), ce qui a eu des répercussions tant nutritionnelles que sociales, néfastes (Chaulet, 1991). Toutefois le niveau de consommation, privilégié par l'aide, est le plus élevé du Maghreb (tableau 3). Récemment, en raison des limites de la politique du "tout importation", l'élevage bovin a été encouragé, grâce à une augmentation du prix du lait local et une réduction de la subvention sur la poudre importée (Bedrani et al., 1997) (tableau 4). L'Algérie connaît ainsi une recrudescence de "mini-laiteries ", qui tente de canaliser le lait cru. Mais leur création récente ne permet pas d'en juger l'effet sur la production et la collecte. L'Algérie a aussi fortement misé sur des importations de bovins laitiers. Leurs performances zoOtechniques demeurent limitées (Madani et Far, 2002). Au plan de la transformation, le tissu a toujours été étatique. Seul le lait collecté par de petits "laitiers artisans " dans les exploitations suburbaines est revendu en l'état ou transformé. Une différenciation récente entre produits locaux et produits importés émerge, étant donné l'image de qualité que véhiculent avec les produits issus de lait reconstitué. Au Maroc, les choix opérés ont été radicalement différents. L'élevage bovin a été promu et la poudre de lait importée fortement taxée. Un dispositif d'aide à la production et à la collecte du lait, a permis aux usines de transformation de s'approvisionner sur place. Le lait provient d'une kyrielle de types d'élevages (fermes spéle lait frais et ses dérivés, par comparaison cialisées, unités paraétatiques, exploita- tions paysannes...), qui ont été séduits par une politique de prix attractifs. En revanche, le prix à la consommation est demeuré élevé, freinant les niveaux de consommation (tableau 5). Le Maroc a aussi importé massivement des génisses laitières (près de 300000 depuis 1970).
Cette opération a eu des résultats certes spectaculaires, mais qui doivent être soumis à critique (Sraïri et Baqasse, 2000), tant au niveau de la carrière des vaches que de leurs performances. L'interdiction de ces importations à la suite de la crise de l'encéphalopathie spongiforme bovine (ESB), entre 2000 et 2004, a mis en relief leur importance, les éleveurs ne maitrisant pas la préparation du renouvellement (Sraïri et Faye, 2004). Au plan de la collecte, l'expérience marocaine s'est caractérisée par une augmentation marquée du nombre de centres coopératifs (de 4 en 1970 à plus de 900 en 2005). Mais, rançon du succès, certains ne présentent pas les conditions de viabilité financière pour assurer les fonctions auxquelles ils se destinaient : outre la collecte du lait, la fourniture d'intrants, voire l'appui technique aux éleveurs (Sraïri et Medkouri, 1998). Cela aggrave leurs défaillances de gestion, se répercutant sur la rémunération des adhérents. Plus en aval, le tissu industriel a été dès le départ privé, construit autour de sociétés et de coopératives. Mais, de fait, une seule

\section{Tableau 3. Structuration de la consommation de lait et de dérivés en l'an $\mathbf{2 0 0 0}$ dans les pays du Maghreb (en millions de litres d'équivalent lait).}

Table 3. Structure of milk and derivatives consumption in the Maghreb countries for the year 2000 (in millions of milk equivalent litres).

\begin{tabular}{lccc}
\hline & Algérie & Maroc & Tunisie \\
\hline & & & \\
Production nationale & 140 & 900 & 670 \\
dont lait usiné & 110 & 485 & 390 \\
Importations & 240 & 960 & 75 \\
Consommation totale & 3380 & 1860 & 745 \\
Taux de couverture (\%) & 34 & 58 & 90 \\
Prix du lait à la consommation (euros) & 0,34 & 0,53 & 0,49 \\
Consommation par habitant (kg/an) & 113 & 38 & 75 \\
\hline
\end{tabular}

\section{Tableau 4. Environnement humain et performances laitières en Algérie de 1970 à 2000.}

Table 4. Human environment and dairy performance in Algeria from 1970 to 2000.

\begin{tabular}{lcccc}
\hline \multicolumn{1}{c}{ Année } & $\mathbf{1 9 7 0}$ & $\mathbf{1 9 8 0}$ & $\mathbf{1 9 9 0}$ & $\mathbf{2 0 0 0}$ \\
\hline Population humaine (en millions) & 13,7 & 18,7 & 22,4 & 30,6 \\
Population bovine totale (en millions) & 885 & 1363 & 1393 & 1595 \\
Production laitière (en millions de litres) & 289 & 518 & 630 & 1039 \\
Prix du lait à la ferme (en dinars) - (1) & 0,62 & 2,00 & 10,00 & 22,00 \\
Prix du lait à la consommation (en dinars) - (2) & 0,85 & 1,30 & 2,00 & 20,00 \\
(1)/(2) x 100 (en \%) & 72,9 & 153,8 & 500,0 & 110,0 \\
\hline
\end{tabular}

1 euro $=94,46$ dinars algériens . 
Tableau 5. Environnement humain et performances laitières au Maroc de 1970 à 2000.

Table 5. Human environment and dairy performance in Morocco from 1970 to 2000.

\begin{tabular}{lcccc}
\hline \multicolumn{1}{c}{ Année } & $\mathbf{1 9 7 0}$ & $\mathbf{1 9 8 0}$ & $\mathbf{1 9 9 0}$ & $\mathbf{2 0 0 0}$ \\
\hline Population humaine (en millions) & 15,3 & 20,4 & 24,0 & 29,7 \\
Population bovine totale (en millions) & 3,60 & 3,38 & 3,35 & 2,68 \\
Production laitière (en millions de litres) & 450 & 730 & 895 & 1184 \\
Prix du lait à la ferme (en dirhams) (1) & 0,54 & 1,44 & 2,62 & 2,94 \\
Prix du lait à la consommation (en dirhams) (2) & 1,05 & 2,10 & 4,10 & 5,40 \\
(1)/(2) x 100 (en \%) & 52,9 & 68,6 & 63,9 & 54,4 \\
\hline
\end{tabular}

1 euro $=11,35$ dirhams.

unité assure près de $65 \%$ de la collecte, ce qui la place en position de monopole sur de nombreux bassins laitiers.

Ces dernières années, l'ajustement structurel a induit un désengagement brusque de l'État. Cela génère des transformations profondes au niveau des systèmes d'élevage, d'autant que le décalage entre les prix du lait à la ferme et le prix à la consommation s'est accru sensiblement. La situation de rente croissante se renforce pour les industriels laitiers, qui bénéficient d'un marché protégé. Augmentation de prix des intrants, réduction des marges et difficultés de vendre dans les périodes d'excédents sont des problèmes auxquels sont confrontés les éleveurs. Des flux de lait croissants échappent au circuit formel, surtout aux abords des grands centres urbains (Letheuil, 1999). Dans ces conditions, certains éleveurs s'engagent dans un processus de marginalisation de la production laitière au profit de la viande, même dans des régions a priori favorables (Sraïri et al., 2003).

En Tunisie, les deux approches ont été expérimentées. Après une période durant laquelle la politique a favorisé la reconstitution du lait à partir de poudre importée, une politique d'encouragement de la production et de la collecte locales a été adoptée. Elle est intervenue après l'aggravation du déficit en lait et la hausse de sa facture d'importation (tableau 6). À partir de 1983, une prime correspondant à $20 \%$ du prix payé aux producteurs a été attribuée aux centres de collecte. Mais le décollage effectif ne va avoir lieu qu'à partir du début des années 1990, avec des mesures plus concrètes comprenant les volets suivants (Bourbouze et Elloumi, 1998) :

- instauration d'une surtaxe sur la poudre de lait importée ;

- extension de la subvention à la consommation du lait ;
- augmentation du prix du lait à la production;

- facilités pour l'importation de vaches de races laitières ;

- garantie d'un approvisionnement régulier en aliments concentrés surtout par importations ;

- encouragement des cultures fourragères en sec et en irrigué.

L'ensemble de ces mesures s'est traduit par une augmentation notable de la production de lait frais. Par ailleurs, à la fin des années 1990, une diminution progressive de la protection de la production locale est intervenue (tableau 7), ce qui

\section{Tableau 6. Environnement humain et performances laitières en Tunisie de 1970 à 2000.}

Table 6. Human environment and dairy performance in Tunisia from 1970 to 2000.

\begin{tabular}{lcccc}
\hline \multicolumn{1}{c}{ Année } & $\mathbf{1 9 7 0}$ & $\mathbf{1 9 8 0}$ & $\mathbf{1 9 9 0}$ & $\mathbf{2 0 0 0}$ \\
\hline Population humaine (en millions) & 5,0 & 6,3 & 7,5 & 9,8 \\
Population bovine totale (en millions) & 0,70 & 0,60 & 0,62 & 0,78 \\
Production laitière (en millions de litres) & 140 & 212 & 400 & 870 \\
Prix du lait à la ferme (en dinars) (1) & 0,054 & 0,130 & 0,290 & 0,360 \\
Prix du lait à la consommation (en dinars) (2) & 0,084 & 0,100 & 0,320 & 0,650 \\
(1)/(2) x 100 (en \%) & 64,3 & 130,0 & 90,6 & 55,4 \\
\hline
\end{tabular}

1 euro $=1,76$ dinar tunisien

\section{Tableau 7. Évolution des taux de protection du lait en Tunisie (en \%) (d'après Centre international des hautes études agronomiques méditerranéennes et Institut de la recherche et de l'enseignement supérieurs agricoles, 2002).}

Table 7. Variation in milk protection rates in Tunisia (\%) (from Centre international de hautes études agronomiques méditerranéennes et Institut de la recherche et de l'enseignement supérieurs agricoles, 2002).

\begin{tabular}{lcccccc}
\hline Année & $\mathbf{1 9 9 5}$ & $\mathbf{1 9 9 6}$ & $\mathbf{1 9 9 7}$ & $\mathbf{1 9 9 8}$ & $\mathbf{1 9 9 9}$ & $\mathbf{2 0 0 0}$ \\
\hline & & & & & & \\
TPN $^{*}$ & 55,8 & 62,2 & 21,4 & 19,8 & 15,3 & 3,6 \\
TPE $^{*}$ & 128,7 & 146,8 & 40,7 & 38,2 & 28,9 & 8,3 \\
\hline
\end{tabular}

*TPN : taux de protection nominale ; **TPE : taux de protection effective. lui assure un débouché sur un marché agronomiques par habitant reste cependant bas, même

\section{Performances}

\section{des filières laitières dans les pays
du Maghreb}

La production laitière dans les trois pays a connu un net accroissement (figure 1) augmentation (Bourbouze et al, 1989). témoignant surtout de l'impact des sécheirrigation n'arrive pas à modérer En Algérie, les mesures d'ajustement structurel ont imposé la baisse du niveau 


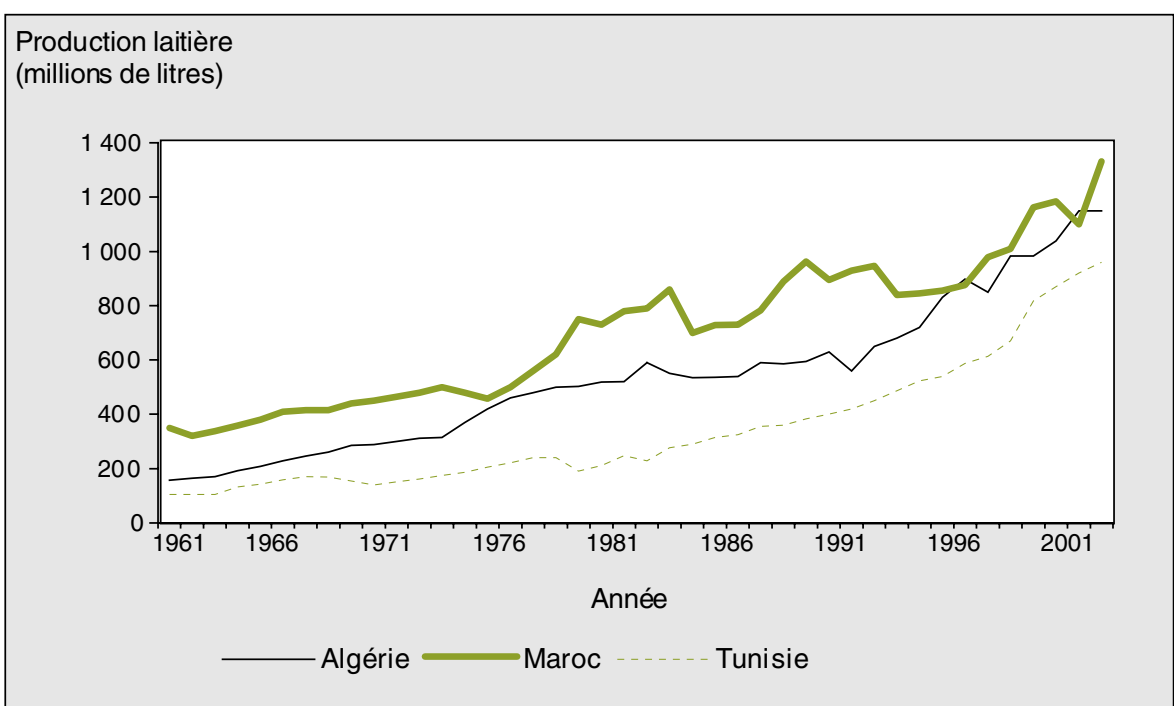

Figure 1. Évolution de la production laitière bovine dans les pays du Maghreb (en millions de litres) (d'après FAO, 2003).

Figure 1. Variation of cattle milk production in the Maghreb countries (million of litres) (from FAO, 2003).

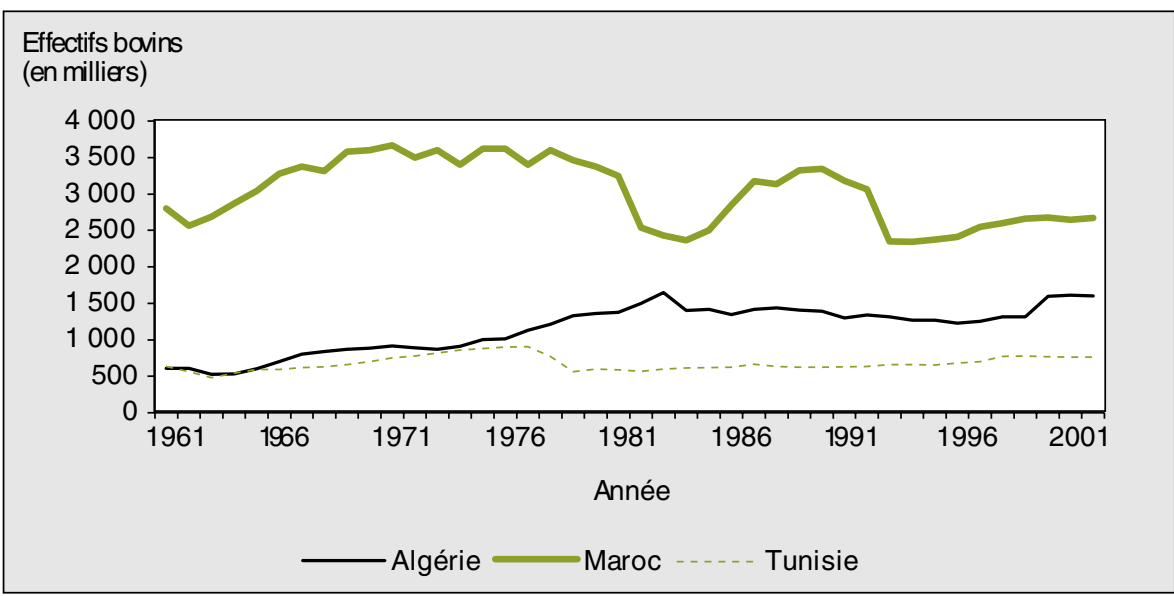

Figure 2. Évolution de la taille du cheptel bovin dans les pays du Maghreb (en milliers) (d'après FAO, 2003).

Figure 2. Variation of total cattle population in the Maghreb countries (thousands) (from FAO, 2003). en 1970 à $55 \%$ en 2005. Au cours de la même période, la superficie emblavée en fourrages est passée de 118000 à 442000 hectares. Un élément clé dans la politique laitière a été l'irrigation: les $12 \%$ de surface agricole utile (SAU) du pays en irrigué assurent près de $45 \%$ de la production. Malgré ces évolutions, les étables laitières sont majoritairement de petites structures (moins de 10 hectares et 5 vaches par étable) (Ministère de l'Agriculture et du Développement rural, 2003). Les stratégies de production s'appuient autant sur la viande (élevage et engraissement des veaux) que sur le lait (Sraïri et al., 2003). La moyenne annuelle de production par vache laitière (tout type génétique confondu) reste faible (inférieure à $2000 \mathrm{~kg}$ ) et profondément marquée par l'aléa climatique (Sraïri et El Khattabi, 2001). Se greffe sur cela la stagnation du prix du lait à la ferme, qui remet en cause les options originelles de développement de l'élevage bovin au Maroc (Kydd et Thoyer, 1993).

En aval, la consommation de produits laitiers n'a pas connu d'amélioration sensible. Les niveaux moyens de consommation (38 kg équivalent de lait par habitant et par an), très inférieurs aux normes nutritionnelles internationales, se caractérisent aussi par des disparités entre urbains et ruraux : 52 et $18 \mathrm{~kg}$ respectivement (Haut commissariat au plan, 2003).

En Tunisie, la filière laitière a aussi connu diverses étapes d'évolution. De 1970 à 1985, la production laitière n'a crû qu'à un rythme annuel moyen de $4 \%$ contre $6 \%$ pour la période 1985-1994. À la fin des années 1980, le cheptel de race laitière représentait à peine le quart de la population bovine. Dans les meilleures conditions, les niveaux de performances demeuraient faibles (Rondia et al., 1985). Les mesures adoptées à la fin des années 1980 ont abouti à l'instauration d'une de subvention des produits laitiers importés (Ait Amara, 1991). La conséquence a été une chute de consommation per capita de 119 à 95 kg/an entre 1988 et 1996 . La production intérieure continue de souffrir de handicaps majeurs, notamment l'insuffisance de fourrages. De surcroit, les succès escomptés en matière d'approvisionnement avec du lait local risquent de ne pas aboutir, car la collecte est difficile : entre 1991 et 2004, elle n'est passée que de 3 à $8 \%$ du volume produit (tableau 8).

Au Maroc, la part des bovins de race locale a chuté de $90 \%$ des effectifs totaux

\section{Tableau 8. Évolution de la collecte de lait cru et de son taux} d'intégration de la production totale en Algérie (en millions de litres).

Table 8. Raw milk collection and its level of integration into the total domestic production in Algeria (in millions of litres).

\begin{tabular}{cccc}
\hline Année & $\begin{array}{c}\text { Production totale } \\
\text { de lait cru }\end{array}$ & Collecte & $\begin{array}{c}\text { Taux de collecte } \\
\text { (\%) }\end{array}$ \\
\hline 1991 & 1156 & 38,6 & 3,3 \\
1995 & 1466 & 125,0 & 8,5 \\
2000 & 1584 & 100,8 & 6,4 \\
2004 & 1782 & 140,3 & 7,9 \\
\hline
\end{tabular}

Source: données statistiques de la Direction de la régulation du développement des productions animales (DRDPA) Alger (2005). 
filière plus organisée et à la modification de la structure génétique du cheptel. Par conséquent, une croissance importante et soutenue de la production a été enregistrée (de 400 à 730 millions de litres entre 1990 et 1998) (Lahmar et al., 2005), conjuguée à une extension du réseau de collecte, une augmentation de la capacité de transformation et une diversification de la gamme des produits transformés. Toutefois, à partir de 2001-2002, la production a décru, en raison de la succession d'épisodes de sécheresse, mettant en relief la forte dépendance vis-à-vis des aléas climatiques. L'augmentation des effectifs de vaches laitières spécialisées est due en grande partie à l'implantation d'élevages bovins dans des régions qui ne s'y prêtent pas vraiment (oasis et ceintures urbaines). Dans ces situations en " hors-sol ", les performances de productivité des vaches et la qualité du lait sont en deçà des standards (Lahmar et al., 2003). Aussi, des marges importantes d'amélioration de la productivité par vache demeurent-elles possibles (Lachaal et al., 2003), tout comme se profilent des actions importantes à mener dans le domaine de la qualité.

\section{Perspectives de développement des filières laitières au Maghreb}

Les filières laitières du Maghreb ont d'indéniables enjeux à relever. Le plus vital est relatif à la sécurisation de l'approvisionnement de populations qui expriment de légitimes aspirations à une meilleure alimentation lactée. Aussi, les projections d'augmentation de la production, doivent-elles s'accompagner de politiques ciblées. Avec le désengagement des États, le recours à des décisions courageuses aux conséquences douloureuses (disparition d'étables, promotion de systèmes d'élevage durables, etc.) est de mise. La réflexion sur le choix de races adaptées demeure aussi ouverte. En effet, la race Holstein ne semble pas avoir été une panacée, surtout pour les élevages paysans à la trésorerie limitée. L'option de races rustiques (comme la Tarentaise, la Simmental ou la Fleckvieh) pourrait s'avérer intéressante. La sélection des races locales doit aussi être envisagée, étant donné leur poids dans les effectifs, la qualité de leurs produits (des taux butyreux supérieurs à $50 \mathrm{~g} / \mathrm{kg}$ ), et leur adaptation aux disettes. La valorisation de l'eau d'irrigation sera à l'avenir un facteur primordial pour la compétitivité du lait par rapport à d'autres spéculations. Jusqu'ici l'élevage bovin s'est implanté sans considération pour la valorisation de l'eau, jouant de ses avantages sociaux (revenu quotidien, emplois, protéines de haute valeur biologique...) pour s'imposer par rapport aux cultures. Mais cela peut s'avérer insuffisant dans des situations de sécheresse. L'aridité qui sévit au Maghreb amène aujourd'hui les décideurs à remettre en question le prix de l'eau et les modalités de sa distribution, ce qui devrait induire une augmentation des prix de revient du lait. Dans ce même registre, la suppression des subventions aura des conséquences sur l'équilibre économique des élevages bovins. Plus en aval, la mise à niveau des industries laitières maghrébines, associées à des multinationales, va imposer l'émergence du discours relatif à la qualité. Or, il est notoire que jusqu'à présent, seuls les aspects quantitatifs ont primé, en accord avec les priorités d'antan. Les références sur la qualité du lait et ses liaisons aux pratiques sont encore rarissimes et témoignent de grandes lacunes, avec une charge microbienne très élevée (Sraïri et al., 2005). Intervenir à ce niveau, c'est se doter d'outils de distinction des fluctuations, surtout à l'amont où l'offre est atomisée et où des agrégations précoces de lots interviennent. C'est aussi établir un système de rémunération équitable qui favorise l'émulation et sanctionne les fautifs et fraudeurs

Il convient aussi de raisonner les termes de logistique de collecte du lait, car de nombreux industriels s'activent dans des bassins de taille incongrue. Cela, à l'image des surcoûts liés à la "nonqualité ", grève leurs revenus. Il est aussi crucial de veiller à l'émergence de débats sereins et continus, autour des enjeux vitaux de la filière : volumes, qualité, saisonnalité, primes, sanctions, redistribution du revenu... Ainsi, un des outils de base pour la gestion des affaires de la qualité serait la constitution de laboratoires indépendants, comme ceux qui sont opérationnels dans les pays avec une interprofession laitière active. Or, la situation actuelle est éloignée de ce schéma, car les concertations entre les opérateurs des filières laitières sont encore rares, avec des rapports de force déséquilibrés entre des milliers d'éleveurs éparpillés et quelques industriels puissants.

Un autre enjeu majeur des filières laitières maghrébines est lié à leur compétitivité, en raison de l'imminence de l'ouverture des marchés aux produits importés. Certes, le lait demeure un produit ardu à commercialiser en raison de sa teneur élevée en eau et des coûts énergétiques de son assèchement et transport : à peine $7 \%$ de la production mondiale est exportée (Onilait, 2005). Toutefois, rien n'indique que la baisse des taxes sur la poudre importée ne la rende pas plus intéressante que le lait local, d'autant que la qualité de ce dernier laisse à désirer.

\section{Conclusion}

L'analyse des filières laitières maghrébines révèle des évolutions contrastées, suite aux différentes politiques mises en œuvre. L'expérience marocaine, et plus récemment, l'expérience tunisienne ont privilégié un approvisionnement local par du lait frais, à la différence de l'Algérie qui, a surtout eu recours aux importations de poudre. En dépit de ces différences, les performances de productivité et de qualité demeurent limitées, marquées par la faiblesse du savoir-faire laitier dans les élevages bovins. Par ailleurs, les achats de concentrés et l'irrigation, peinent à contrecarrer les effets des aléas climatiques et du manque de fourrages, ce qui plombe les marges des éleveurs. De même, la réflexion sur les politiques d'amélioration génétique, basées jusqu'ici sur les importations de vaches Holstein, n'a pas encore intégré ni les possibilités d'acclimatation d'autres souches à double finalité ni le poids des races locales. La collecte et la transformation laitière affichent des performances nuancées, car de nombreux opérateurs traitent des volumes réduits, ce qui entrave leurs perspectives de durabilité face à l'ouverture des marchés. La consommation demeure modeste, en raison du prix du lait élevé, eu égard au pouvoir d'achat des consommateurs. Elle n'atteint pas les recommandations internationales et dévoile d'importantes disparités sociales, surtout entre ruraux et citadins. Dans leur globalité, les dynamiques récentes des filières laitières maghrébines sont le fruit d'ajustements qui ont été opérés au cours des trente dernières années, indiquant que les résultats dans ce domaine nécessitent du temps afin de tester effectivement 
sur le terrain les options retenues et d'ajuster leurs résultats. La priorité s'oriente vers la sécurisation de l'approvisionnement des populations et aussi vers le maintien d'un élevage bovin rémunérateur pour les milliers d'exploitations qui le pratiquent. Aussi, les pouvoirs publics et les acteurs des filières laitières maghrébines sont-ils en pleine réflexion sur la mise à niveau du secteur pour atteindre ces objectifs. En amont, cela passe par la localisation de cette activité au sein des régions et des exploitations qui s'adaptent le plus, par les atouts qu'elles affichent. En aval, cela suppose de cerner les facteurs de variation de la qualité du lait, d'optimiser les circuits logistiques de collecte, et de diversifier la gamme des produits pour satisfaire des consommateurs de plus en plus exigeants et pouvant aussi accéder plus facilement à des produits laitiers importés. C'est d'ailleurs ce dernier élément d'ouverture des frontières maghrébines à des produits d'outremer qui constitue un facteur clé avec lequel devront composer les filières des trois pays pour assurer leur pérennité, dans un souci de garantir la paix sociale, la sauvegarde d'emplois et la fourniture de produits à haute valeur nutritionnelle.

\section{Références}

Abaab A, Bédrani S, Bourbouze A, Chiche J. Les politiques agricoles et la dynamique des systèmes agropastoraux au Maghreb. Options Méditerranéennes Ser B 1995 ; 14 : 139-65.

Aït Amara A. Algeria : Agriculture and Indus try. In : Aït Amara A, Founou Tchuigoua B, eds. African Agriculture : the Critical Choices. Coll. "Studies in African Political Economy". United Nations University. Third World Forum. New York : United Nations University, 1991.

Balta P. Le Grand Maghreb: des indépendances à I'an 2000. Paris : éditions de La Découverte, 1990.

Bedrani S, Boukhari N, Djennane A. Éléments d'analyse des politiques de prix, de subvention et de fiscalité sur l'agriculture en Algérie. Options Méditerranéennes Ser B 1997 ; (11): 121-49.

Bencharif A, Chaulet C, Chehat F, Kaci M Sahli Z. La filière blé en Álgérie. Paris : éditions Karthala, 1996.

Bourbouze A, Elloumi M. Mission d'évaluation concernant la coopération francotunisienne dans le domaine de la filière laitière en Tunisie. Montpellier : Institut agronomique méditerranéen de Montpellier (lamm), 1998.

Bourbouze A, Chouchen A, Eddebbarh A, Pluvinage J, Yakhlef $\mathrm{H}$. Analyse comparée de I'effet des politiques laitières sur les structures de production et de collecte dans les pays du Maghreb. Options Méditerranéennes Ser Seminaires $1989 ;(6): 247-58$.
Chalmin P. Cyclope. Lait et produits laitiers. Paris : éditions Économica, 1999.

Chaulet C. Agriculture et nourriture dans les réformes algériennes : un espace pour les paysans. Rev Tiers Monde 1991 ; XXXII : 741-70.

Centre international des hautes études agronomiques méditerranéennes (Ciheam) : Institut de la recherche et de l'enseignement supérieurs agricoles (Iresa). Le soutien aux produits agricoles et aux filières agro alimentaires, études de cas: Maroc, Tunisie Turquie. Montpellier: Institut agronomique méditerranéen de Montpellier (lamm), 2002.

Djemali M, Berger PJ. Yield and reproduction characteristics of Friesian cattle under North African conditions. J Dairy Sci $1992 ; 75: 3568$ 75.

Dufumier M. Sécurité alimentaire et systèmes de production agricole dans les pays en développement. Cah Agric 1996 ; 5 : 229-37.

El Khyari T. Agriculture au Maroc. Casablanca : éditions Okad, 1985.

Faye B, Bengoumi M, Hidane K. Le développement de l'élevage laitier camelin péri-urbain l'exemple de Laâyoune (provinces sahariennes du Maroc). In : Duteurtre G, Meyer C, eds. Marchés urbains et développement laitier en Afrique sub-saharienne. Montpellier: Cirad édtions, 1998.

Food and Agriculture Organisation (FAO). FAO stastistical databases. Rome : FAO, 2003.

Guessous F. Production fourragère et systèmes animaux. Rabat: Actes éditions; Institut agronomique et vétérinaire Hassan II (IAV Has san II), 1991.

Haut Commissariat au Plan (Royaume du Maroc). Enquête nationale de Consommation des Ménages. Rabat: Haut Commissariat au Plan, 2003.

Jaritz G, Bounejmate M. Production et utilisa tion des cultures fourragères au Maroc. Rabat: Inra éditions, 1995.

Kydd J, Thoyer S. Structural adjustment and Moroccan Agriculture : an assessment of the reforms in the sugar and cereal sectors. Paris : éditions de I'OCDE, 1993.

Lachaal L, Lahmar L, Gharbi F, Bouraoui R. A stochastic frontier analysis of technical efficiency for dairy production in Northern Tunisia. Proceedings of the International Symposium "Prospects for a sustainable dairy secto in the Mediterranean". EAAP Publication n'99. Wageningen (The Netherlands) : Wageningen Publications, 2003.

Lahmar M, Djemali M, Khemiri H. Cattle production in the mountain regions of North West Tunisia: present situation and prospects for increased productivity. Proceedings of the International Symposium "Animal Production and Natural Resources Utilisation in the Mediterranean Mountain Areas". EAAP no 115 Wageningen (The Netherlands) : Wageningen Publications, 2005.

Lahmar M, Bouraoui R, Lachaal L, Toumi J. Characterisation of milk production systems in Tunisia: the case of Ariana region. Proceedings of the International Symposium "Prospects for a sustainable dairy sector in the Mediterranean". EAAP Publication n99. Wageningen (The Netherlands) : Wageningen Publications, 2003.
Letheuil H. La filière lait à Casablanca : approvisionnement, typologie des unités de transformation, commercialisation. Mémoire de $3^{e}$ cycle, Centre national d'études agronomiques des régions chaudes (Cnearc), Montpellier, 1999.

Madani T, Far Z. Performances de races bovines laitières améliorées en région semi-aride algérienne. Neuvièmes rencontres recherches ruminants. Paris: Inra; Institut de l'élevage, 2002.

Ministère de I'Agriculture et du Développement rural (MADR). Statistiques de la situation de l'élevage et de l'agriculture au Maroc. Rabat : MADR, Direction de I'élevage, 2003.

Office national interprofessionnel du lait et des produits laitiers (Onilait). Commission permanente des marchés : bilan 2004 et perspectives 2005. Paris: Onilait, 2005. http ://www.oni lait.fr/Documents/EMP-bilan2004persp2005.pdf.

Projet Sebou. Mise en valeur agricole de la plaine du Gharb. Fascicule 4, Livret 2. Rabat : Ministère de I'Agriculture et de la réforme agraire, 1961.

Rondia G, Deker A, Jabari M, Antoine A. Produire plus de grain et de lait en Afrique du Nord. Projet de ferme modèle de Frétissa. Rapport final. Gembloux (Belgique) : Faculté des sciences agronomiques de Gembloux, 1985.

Spedding CRW. An introduction to agricultural systems. 2nd edition. London: Elsevier Applied Science, 1979.

Sraïri MT, Faye B. Pratiques d'élevage de bovins laitiers considérées à l'aune du discours technique: quelques exemples à partir du Maroc. Ethnozootechnie 2004 ; 74 : 47-58.

Sraïri MT, El Khattabi M. Évaluation économique et technique de la production laitière intensive en zone semi-aride au Maroc. Cah Agric 2001; $10: 51-6$.

Sraïri MT, Baqasse M. Devenir et performances de génisses pie noires frisonnes importées au Maroc. Livestock Research for Rural Development $2000 ; 12$ : 3. http ://www.cipav.org. co./IIrd/IIrd12/3/ sra123 htm.

[Consulté le 10/12/06].

Sraïri MT, Ilham A. L'élevage bovin laitier en zones irriguées et sa sensibilité à I'aléa climatique : cas du Maroc In: Guessous F, Rihani N, llham A, eds. Livestock production and climatic uncertainty in the Mediterranean. EAAP $n^{\circ}$ 94. Wageningen (The Netherlands) : Wageningen Publications, 2000.

Sraïri MT, Medkouri H. Production et écoulement du lait en région d'agriculture pluviale au Maroc. Tropicultura 1998 : (16-17) : 321-6.

Sraïri MT, Leblond JM, Bourbouze A. Production de lait et/ou de viande: stratégies des éleveurs de bovins dans le périmètre irrigué du Gharb au Maroc. Rev Elev Med Vet Pays Trop $2003 ; 56: 177-86$.

Sraïri MT, Hasni Alaoui I, Hamama A, Faye B. Relations entre pratiques d'élevage et qualité globale du lait de vache en étables suburbaines au Maroc. Rev Med Vet (Toulouse) 2005 $156: 155-62$.

Talha L. Croissance, crise et mutations économiques au Maghreb. In : Bichara K, ed. Alternatives Sud: Ajustement structurel au Maghreb. Vol. II (3). Louvain La Neuve: Éditions du Centre d'études et de recherche du monde arabe contemporain, 1994. 